Tecno Lógicas

ISSN 0123-7799

Vol. 20, No. 38, pp. 95-106

Enero - junio de 2017

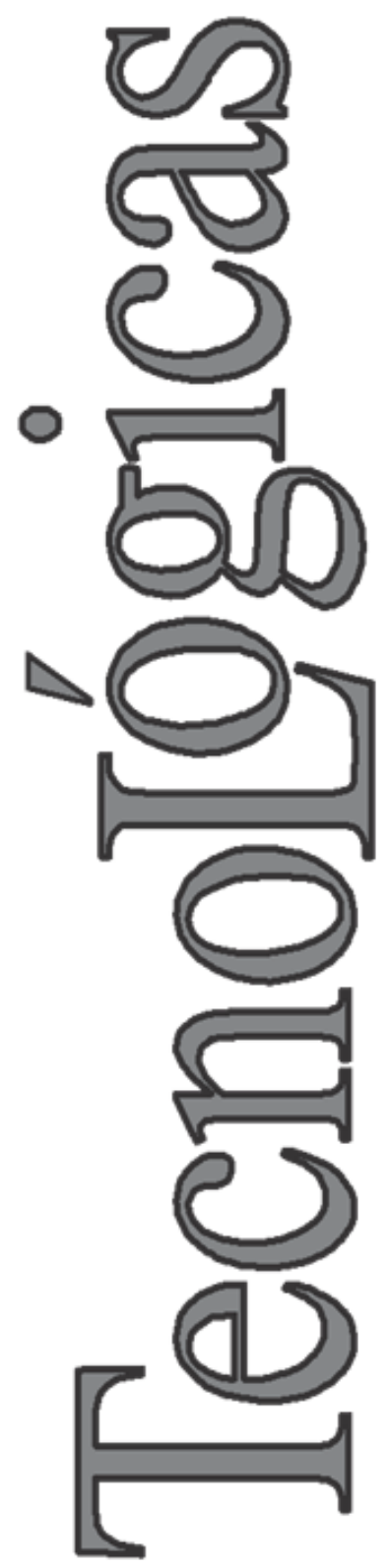

(C) Copyright 2015 por autores y Tecno Lógicas Este trabajo está licenciado bajo una Licencia Internacional Creative Commons Atribución (CC BY)

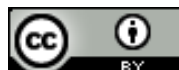

\section{Análisis numérico de la influencia de la granulometría para la generación de flujos secos de detritos mediante el DEM}

\section{Numerical analysis of the grain size distribu- tion in the activation of dry debris flow by means of DEM}

\author{
Gerardo Arévalo-Mendoza ${ }^{1}$, \\ Alfonso M. Ramos-Cañón ${ }^{2}$ y Luis F. Prada-Sarmiento ${ }^{3}$
}

Recibido: 11 de febrero de 2016, Aceptado: 19 de septiembre de 2016
Cómo citar / How to cite

Gerardo Arévalo-Mendoza, Alfonso M. Ramos-Cañón, y Luis Felipe Prada-Sarmiento, "Análisis numérico de la influencia de la granulometría para la generación de flujos de detritos mediante el uso del Método de Elementos Discretos (DEM)", Tecno Lógicas, vol. 20, no. 38, pp. 95-106 enero-junio, 2017.
1 Ingeniero Civil, Departamento de Ingeniería Civil, Pontificia Universidad Javeriana, Bogotá-Colombia, b.arevalo@javeriana.edu.co

2 Doctor en Ingeniería, MSc en Geotecnia, Ingeniero Civil, Instituto Geofísico, Facultad de Ingeniería, Pontificia Universidad Javeriana, Bogotá-Colombia, a-ramos@javeriana.edu.co

3 Doctor en Ingeniería, Ingeniero Civil, Departamento de Ingeniería Civil, Pontificia Universidad Javeriana, Bogotá-Colombia, lf.pradas@javeriana.edu.co 


\section{Resumen}

El flujo de detritos es un proceso de naturaleza granular que ha sido abordado ampliamente bajo metodologías basadas en el medio continuo. Sin embargo, estas aproximaciones simplifican la realidad granular del suelo. Como material granular, la granulometría puede gobernar el comportamiento de este movimiento desagregado de masa. Es posible estudiar este comportamiento en términos del ángulo máximo alcanzado $(\theta)$, la energía cinética (Ek) y la profundidad de flujo ( $\mathrm{Fd}$ ) con el fin de establecer condiciones potenciales de falla, así como sus capacidades de daño. Para abordarlo se hace uso del método de elementos discretos, desarrollado por [1], bajo un estudio paramétrico en el cual se varían los parámetros de la granulometría: el tamaño medio del grano (d50), el coeficiente de curvatura $(\mathrm{Cu})$ y el tamaño máximo de la muestra (dmax). Los resultados muestran que existe una influencia apreciable y una estrecha relación en los parámetros Ek y Fd. Con respecto al $\theta$ alcanzado en superficie se tiene una variación de menos de $5^{\circ}$ para valores extremos de d50. Con esto, se muestra que la granulometría puede ser un factor importante que influye en la generación de flujos de detritos y se encuentran relacionadas cercanamente.

\section{Palabras clave}

Flujo de detritos, granulometría, método de elementos discretos, tambor rotador, profundidad de flujo.

\section{Abstract}

Debris flow is a process of granular nature that has been widely analysed with methodologies based upon continuum mechanics. However, those approaches do not take into account the real granular condition of the soil. Grain size distribution exerts an important control on the movement of debris flows. This behaviour can be studied by analyzing three variables: maximum tilt angle $(\theta)$, kinetic energy (Ek) and flow depth (Fd). These variables allow to obtain a deeper insight into the conditions that trigger the failure of slopes and its subsequent capacity of damage. This research resorts to the use of the discrete element technique, developed by [1], performing a parametrical study of the parameters controlling grain size distributions like: mean size (d50), coefficient of curvature $(\mathrm{Cu})$ and maximum size (dmax). The results showed an important influence and also a strong interaction between Ek and $\mathrm{Fd}$ on the onset of granular flows. The angle $\theta$ reached on the surface has a variation less than $5^{\circ}$ with extreme values of $\mathrm{d} 50$. Results reported herein, allow to recognize the influence of grain size distribution on the triggering of slides of granular materials.

\section{Keywords}

Debris flow, grain size distribution, discrete element method, rotating drum, depth flow. 


\section{INTRODUCCIÓN}

Los deslizamientos son el sexto fenómeno natural relevante por las pérdidas humanas registradas. Las tasas de mortalidad son mayores cuando se generan flujos de detritos, además de generar una seria afectación en la infraestructura como lo reporta [2]. El método de equilibrio límite es el procedimiento más empleado para estudiar los procesos de remoción en masa. Esta metodología presenta varias limitaciones: a. El modo de deformación supuesto no es el que se presenta en la mayoría de las situaciones en campo. b. Supone que la movilización de resistencia se presenta en la totalidad de la superficie potencial de falla. c. Como resultado del método se obtiene un factor de seguridad, pero se excluyen características importantes del movimiento como la velocidad de la masa y distancia a la que se deposita [3], [4]. d. El método supone que el medio es continuo, por lo que las características relacionadas con la granulometría no se pueden considerar en el análisis convencional.

Dentro de los deslizamientos, los flujos de detritos tienen una alta tasa de ocurrencia [5] con la particularidad que los daños y pérdidas que pueden ocasionar son altos, dada las grandes extensiones que pueden cubrir. Normalmente estos flujos de detritos ocurren en laderas con altas inclinaciones y valles estrechos [5]. Estos flujos pueden desencadenarse por eventos de lluvia, sismos o pequeñas vibraciones que detonan procesos de inestabilidad en el material. En estos últimos casos, el material puede encontrarse en una condición completamente seca y aun así se genera el movimiento. Los flujos secos de detritos soportan esfuerzos cortantes en condición estática, se pueden deformar lentamente generando cadenas de contactos friccionales entre los granos del material. También pueden moverse a grandes velocidades en donde se generan colisiones rápidas y breves entre los granos del suelo [5]. Cuando aparece el agua, las interacciones de fric- ción y colisiones se ven afectadas por los efectos viscosos y de presión de poros causadas por el fluido intergranular, generando transiciones de momento entre las fases. Ensayos de simulación a escala de flujos de detritos en canaletas inclinadas muestran que para materiales predominantemente granulares, en las oleadas de movimiento no se registran presiones de poros [5]. Bajo esta condición de analizar flujos secos de materiales granulares desagregados sin considerar efectos de presión de poros ni viscosidad del fluido se plantea esta investigación numérica basada en la técnica de los elementos discretos con el fin de analizar la influencia de la granulometría del material en la energía cinética al inicio de la fase de movimiento en un ensayo de tambor rotador.

Se sabe que la granulometría del material tiene una fuerte influencia en las características de un flujo de material desagregado. Sin embargo, la revisión bibliográfica indica que no hay reportes de estudios comprensivos donde se analice la influencia del tamaño máximo, coeficiente de uniformidad y otros indicadores dados por la naturaleza discreta que conforman el material en las características del flujo. El Método de Elementos Discretos (DEM) surge como alternativa para modelar avalanchas ante las limitaciones que impone la teoría del medio continúo y los métodos de equilibrio límite al no considerar la naturaleza particulada del material granular [6]. El DEM tiene la ventaja de considerar explícitamente la interacción de las partículas en un material granular implementando modelos constitutivos de contacto [7].

La generación de flujos de material desagregado se puede estudiar por medio de experimentos físicos y su simulación con DEM en trabajos de análisis del proceso de flujo de detritos acoplado con fluidos [8][10], casos de estudio de eventos reales [11], [12], estudio numérico de la segregación el proceso de flujo de detritos en canales [13] o en particular con el modelo del 
tambor rotativo. En este último experimento, el material granular se recircula generando un proceso continuo y relativamente fácil de comparar con modelos de laboratorio. Este sistema se ha utilizado en estudios del análisis del régimen de flujo en avalanchas por [14]-[18], procesos de segregación [10], [14], [19], simulaciones quasi-reales en tiempo en programación en paralelo [20], evaluación de los efectos de las condiciones geométricas del tambor rotador [21], [22] y el estudio de mezclado de partículas húmedas en la industria química [23], principalmente.

En este trabajo se realizó el estudio del comportamiento del flujo de detritos secos considerando la distribución granulométrica, el cual puede entregar herramientas para comprender la física que detona estos eventos. Este estudio se apoya en la metodología del DEM mediante la simulación del modelo del tambor rotativo.

\section{METODOLOGíA}

\subsection{Método de elementos discretos}

El Método de Elementos Discretos (DEM) fue desarrollado por Cundall y Strack [1] y permite explicar el comportamiento mecánico de materiales granulares a partir de la representación de dichos materiales por medio de geometrías planas de discos (en 2D) o esferas (en 3D). El método hace el seguimiento de cada partícula y sus interacciones con partículas vecinas, descritas por medio de modelos de contacto, que permiten realizar el análisis de los cuerpos que se encuentran en contacto estático o dinámico [24]. [1] hacen uso del desarrollo de Hertz (Fig. 1) para introducirlo como modelo constitutivo elástico no lineal, representativo de la fuerza normal generada en un punto de contacto entre dos esferas. Por otro lado, en la dirección tangente (o de corte) se configura el modelo de la teoría del contacto de fricción elástica, desarrollado por [25].

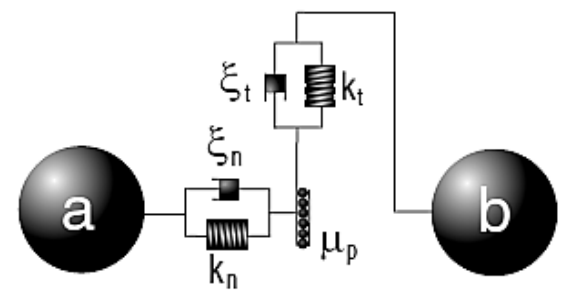

Fig. 1. Modelo de contacto de Hertz- Mindlin. Fuente: [22]

Las expresiones desarrolladas en el modelo de Hertz, se basan en la teoría elástica de dos cuerpos continuos en contacto. De lo anterior se derivan las expresiones de distribución de esfuerzo y deformación entre dos partículas en contacto. Se supone que las partículas exhiben una deformación virtual $(\delta)$ durante el contacto (ver Fig. 2) Esta teoría tiene varias limitaciones: en primer lugar, la rugosidad de la superficie es despreciada (la superficie de contacto es asumida como perfectamente lisa) y las esferas no son capaces de desarrollar fricción [24]. Estas suposiciones son también tomadas por [1], [26], [27] en su implementación de métodos para estudiar el comportamiento de cuerpos discontinuos.

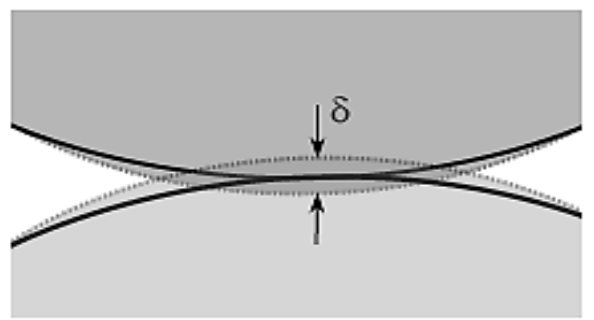

Fig. 2. Modelo de contactos entre cuerpos rígidos. Las líneas continuas indican como debería ser la superficie cuando entra en contacto. Fuente: [24]

La suposición de cuerpos rígidos facilita las simulaciones donde se involucran un gran número de partículas, ya que se supone que el movimiento relativo de las partículas tiene mayor influencia en la respuesta global que las deformaciones de las partículas individuales [7]. Para la deformación de las partículas de manera individual se usa la aproximación de "esfera-suave" [1]. Con esto, se logra reproducir una deformación virtual de la partícula, la 
cual es en realidad un traslapo de magni-

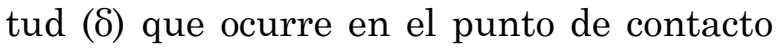
sin deformar las partículas, es decir, conserva su forma perfectamente esférica. La anterior suposición es importante dado que aún en los cuerpos rígidos existe deformación cuando dos partículas entran en contacto [7].

Para representar mejor el comportamiento físico no lineal del suelo, se han desarrollado modelos que permiten relacionar el parámetro de rigidez del resorte con los parámetros de las partículas. Este tipo de modelo de contacto ha sido usado en publicaciones como [22], [23] entre otras. La expresión de la rigidez del resorte en dirección normal (1) y tangencial (2) del modelo de Hertz está dada por:

$K_{n}=\frac{2 G \sqrt{(2 R)}}{3(1-v)} \sqrt{ } \delta_{n}$

$K_{s}=\frac{2\left(G^{2} 3(1-v) R\right)^{1 / 3}}{2-v}\left|F_{n i}\right|^{1 / 3}$

donde $\delta$ n es el traslapo para dos esferas en contacto ( $\mathrm{y} \mathrm{y} \mathrm{b}$ ), donde $\mathrm{R}$ es el radio equivalente, $\mathrm{G}$ es el módulo de corte y $v$ es el coeficiente de Poisson.

En teoría, la resistencia friccional no puede desarrollarse en el contacto entre dos esferas suaves perfectamente esféricas. Sin embargo, dentro de los códigos de DEM se introduce un parámetro de deslizamiento friccional que es asumida por el entrabamiento mecánico entre las asperezas de la superficie rugosa de las partículas de suelo es representada bajo el parámetro friccional que cumple la ley de Coulomb (3):

$\tau=\mu \sigma$

\subsection{Modelo numérico: conformación de la muestra y etapas de simulación}

La distribución de partículas granulares en el tambor rotador se genera aleato- riamente en toda el área del tambor como "agregados" con relación de aspecto 1:1.5 (diámetro de la partícula: longitud del agregado de dos partículas esféricas con traslapo). El tambor rotador se llena mediante la caída por gravedad de las partículas para llenar el $40 \%$ del diámetro del tambor. El porcentaje de llenado del tambor se garantiza con un proceso de corte horizontal (es decir que las partículas por encima de dicho límite son eliminadas dentro del programa). Finalmente, se estabiliza la configuración de las partículas durante 2 segundos en la simulación (etapa A Figura 4), para minimizar los efectos de la carga que ha sido removida. La velocidad de rotación del tambor se fijó en 0.05 RPM. Sin embargo, a esa velocidad se incurriría en tiempos de simulación exageradamente altos [28], [29]. La solución propuesta para controlar el tiempo de simulación es imponer una velocidad de rotación de 10 RPM en el inicio de la simulación, hasta alcanzar un ángulo de $23^{\circ}$ aproximadamente (etapa B), después estabilizar la muestra en 1.3 segundos usando como criterio la "fuerza promedio no balanceada" en un valor de 0.005 (etapa C). Finalmente se impone una velocidad 0.05 RPM durante 8 segundos (etapa $\mathrm{D})$.

\subsubsection{Geometría del tambor}

Dentro de las condiciones de simulación se encuentra la geometría del tambor, la cual está condiciona por 2 dimensiones: la longitud (L) y el diámetro (D) (ver Fig. 3).

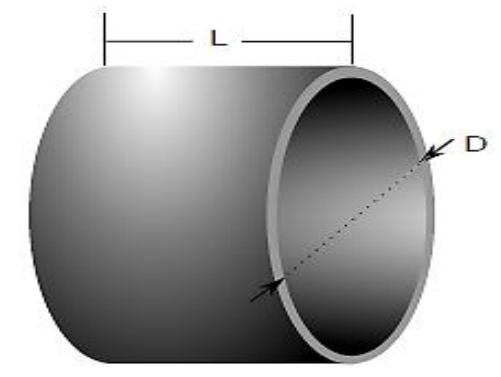

Fig. 3. Dimensiones principales de la geometría del tambor rotador. Fuente: Autores. 
La dimensión de la longitud del tambor no se ve afectada debido a que los muros laterales no tienen fricción. Aunque la longitud no se ve afectada, existen variaciones en las mediciones de energía cinética para la dimensión del diámetro y el volumen de control dentro del tambor rotador. Por otra parte, se detectó que la profundidad de flujo (ver Fig. 4 y Fig. 5) no se ve afectada por las diferentes relaciones de diámetro evaluadas.

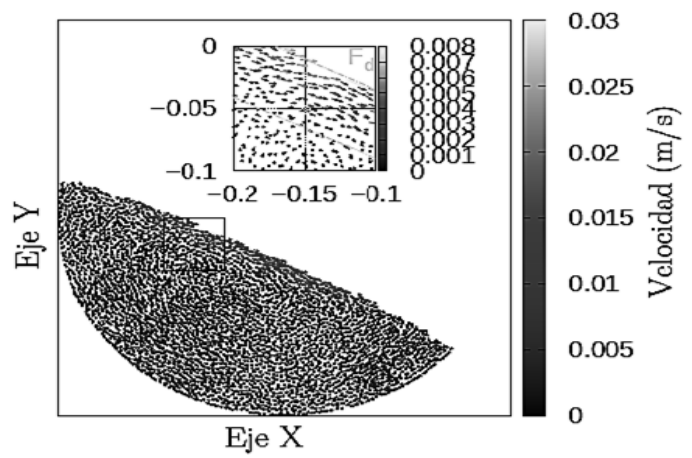

Fig. 4. Energía cinética $\mathrm{D} / \mathrm{d}_{\max }=60$. Fuente: Autores.

Como se observa en la Fig. 4 durante el proceso se genera una profundidad de flujo (Fig. 5 (a)) que puede ser calculada mediante la detección de la magnitud de la velocidad menor. Para ello se normaliza la velocidad y se calcula el punto de inflexión como se observa esquemáticamente en la Fig. 5 (b).
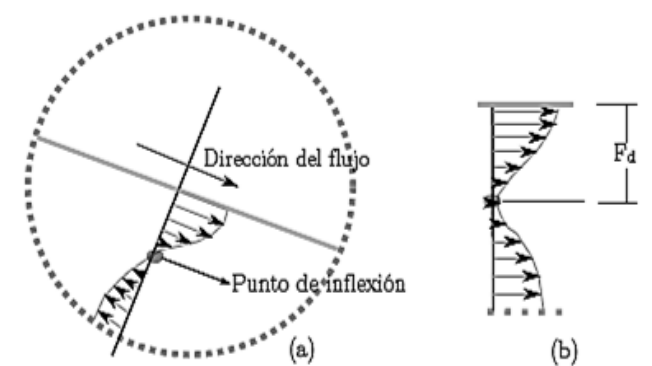

(b)

Fig. 5. Profundidad de flujo: a) Durante el proceso. b) Cálculo. Fuente: Autores.

\subsection{Granulometría}

Una muestra granular natural está generalmente compuesta por diferentes tamaños de partículas que puede ser agru- pado en porcentajes dentro de un rango de tamaños. La gradación de un material granular es el contenido de diferentes tamaños de grano, y este es identificado por el porcentaje de un tamaño especifico de los granos de la muestra [30]. La clasificación de suelos granulares se basa en el coeficiente de curvatura (Cc), coeficiente de uniformidad $(\mathrm{Cu})$, tamaño máximo (dmax) y medio (d50). En el presente trabajo los parámetros estudiados son: $\mathrm{Cu}$, dmax $\mathrm{y}$ d50, cada uno con tres valores diferentes, obteniendo en total 27 combinaciones.

Adicional al d50 y $\mathrm{Cu}$ se evalúa la influencia del dmax en el flujo de detritos. Para este propósito se toman porcentajes a partir del tamaño máximo nominal, es decir el valor de dmax1 es equivalente al 90\% del dmax absoluto. Entonces, los dos valores de tamaño máximo restantes son: $80 \%$ para (dmax 2$)$ y $70 \%$ para (dmax3). Los parámetros granulométricos usados en el estudio se resumen en la Tabla 1.

\begin{tabular}{ccccc}
\multicolumn{5}{c}{ Tabla 1. Valores de $\mathrm{d}_{\max }$} \\
\multicolumn{4}{c}{ usados en las simulaciones. } \\
\hline $\mathrm{C}_{\mathrm{u}}$ & & 1,1 & 2,0 & 4,0 \\
\hline $\mathrm{d}_{50}$ & $\% \mathrm{~d}_{\max }$ & {$[\mathrm{mm}]$} & {$[\mathrm{mm}]$} & {$[\mathrm{mm}]$} \\
\hline & 90 & 5,26 & 7,34 & 10,78 \\
5 & 80 & 5,18 & 6,54 & 8,56 \\
& 70 & 5,12 & 5,96 & 7,12 \\
\hline & 90 & 10,54 & 14,68 & 21,58 \\
10 & 80 & 10,36 & 13,08 & 17,14 \\
& 70 & 10,24 & 11,92 & 14,24 \\
\hline & 90 & 15,80 & 22,02 & 32,36 \\
15 & 80 & 15,56 & 19,64 & 25,72 \\
& 70 & 15,36 & 17,90 & 21,36 \\
\hline
\end{tabular}

\subsection{Parámetros mecánicos}

La selección de los parámetros mecánicos del modelo constitutivo de contacto representa un inconveniente en la modelación micromecánica, dado que no son fáciles de determinar e influyen de manera importante en la respuesta numérica del 
material. La calibración de parámetros es un proceso usado con frecuencia en ingeniería y se basa en la relación entre el comportamiento a gran escala obtenido en laboratorio contra el resultado obtenido con el modelo numérico. Los modelos en DEM deben ser capaces de capturar la respuesta observada en los ensayos. Estos ensayos son pruebas de laboratorio con las cuales se obtienen resultados que se comparan con los resultados de la simulación [7], [31]. Generalmente, en los estudios de calibración se varían los parámetros de entrada del modelo para capturar la respuesta a escala macro [8]. La asignación de parámetros a los modelos de contacto se realizó a través de la realización de un análisis paramétrico de los parámetros en ensayos numéricos de control (ensayos de coeficiente de restitución, ángulo de fricción externa y corte directo). Los ensayos numéricos se desarrollaron con el fin que el resultado de cada uno de ellos tuviera el comportamiento esperado de variables macroscópicas típicas. De acuerdo con la calibración de parámetros realizada, se obtiene una serie de parámetros del modelo que se resumen en la Tabla 2.

\begin{tabular}{lcc}
\multicolumn{3}{c}{$\begin{array}{c}\text { Tabla 2. Parámetros mecánicos del modelo. } \\
\text { (C. F=Coeficiente de fricción). }\end{array}$} \\
\hline Variable & Símbolo & Valor \\
\hline Densidad $\left[\mathrm{kg} / \mathrm{m}^{3}\right]$ & $\mathrm{P}$ & 2650 \\
Módulo de elasticidad [MPa] & $\mathrm{E}$ & 10 \\
Coeficiente de Poisson [-] & $\mathrm{N}$ & 0.3 \\
C.F. estático [-] & $\mu$ & 0.3 \\
Coeficiente de restitución [-] & $\mathrm{A}$ & 0.7 \\
C.F. del cilindro [-] & $\mu_{\mathrm{c}}$ & 0.3 \\
C.F. de las paredes laterales [-] & $\mu_{1}$ & 0.0 \\
\hline
\end{tabular}

\section{RESULTADOS Y DISCUSIÓN}

\subsection{Régimen de flujo a nivel macroscópico}

En la generación y desarrollo del proceso de flujo de detritos se pueden evidenciar comportamientos que están influenciados a lo largo del tiempo por otras variables como la granulometría. El flujo de detritos generado mediante el tambor rotador es un proceso que inicia desde la parte superior de la superficie de la muestra y posteriormente se propaga en la dirección del flujo hasta desarrollarse completamente. Un comportamiento adicional que se presenta en todo el proceso, y aún en todas las simulaciones, es la presencia de una celda de movimiento convectivo que cambia de posición a lo largo de la simulación, esta se mantiene en superficie mientras no se genere movilización de la masa.

Cuando el proceso de remoción se genera, la celda de convergencia se ubica por debajo del flujo, muy cerca del centro del mismo. Estas celdas pueden ser producidas por el cambio de dirección de las partículas. Lo anterior debido a que, por equilibrio, siempre habrá un punto donde su velocidad es muy cercana a cero (0), normalmente cuando existe un cambio de dirección. Este comportamiento es general y no parece estar influenciado por la disposición de la muestra o su gradación. La gradación comienza a jugar un papel importante en el desarrollo del flujo de detritos. Como se logra ver en todos los resultados, el comportamiento de las variables como el ángulo máximo (ver Fig. 6), la energía cinética (ver Fig. 7) o la profundidad de flujo (ver Fig. 8) varían a lo largo del tiempo de simulación. Independientemente de la variación en el tiempo, estas variables evaluadas se encuentran estrictamente relacionadas (sombreado verde en las figuras anteriormente mencionadas); sin embargo, el proceso se refleja claramente en las gráficas de energía cinética. Se observa que con valores menores de $d_{50}$ el tiempo de duración del flujo es mayor (alrededor de los 2 segundos) como se muestra en las Fig. 9, Fig. 10 y Fig. 11. A medida que el valor medio de la partícula incrementa, el tiempo de flujo es bastante localizado y se desarrolla en un corto tiempo (alrededor de 1 segundo). 


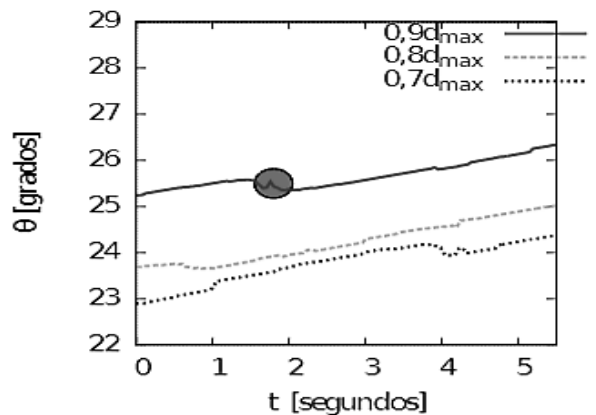

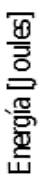

Fig. 6. Evolución del ángulo para un $\mathrm{C}_{\mathrm{u}}=1.1 \mathrm{y} \mathrm{d}_{50}=10 \mathrm{~mm}$. Fuente: Autores.

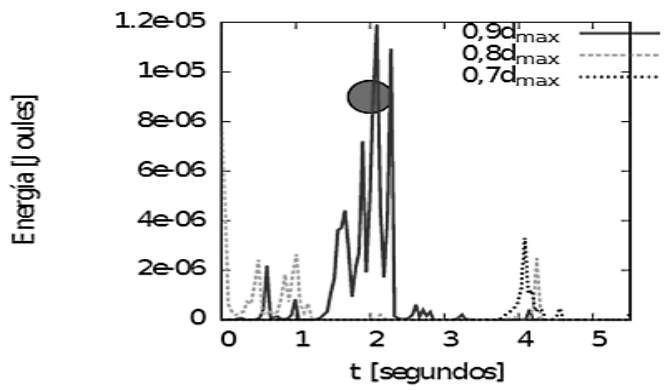

Fig. 7. Evolución de la energía cinética en el tiempo para un $\mathrm{C}_{\mathrm{u}}=1.1$ y d $\mathrm{d}_{50}=10 \mathrm{~mm}$. Fuente: Autores.

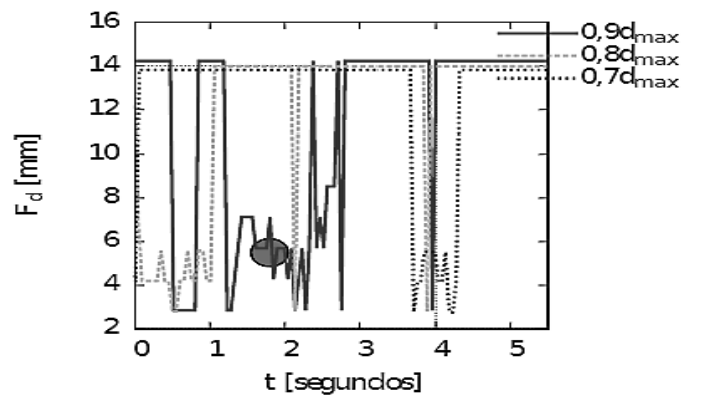

Fig. 8. Evolución de la profundidad de flujo en el tiempo para un $\mathrm{C}_{u}=1.1$ y d ${ }_{50}=10 \mathrm{~mm}$. Fuente: Autores.

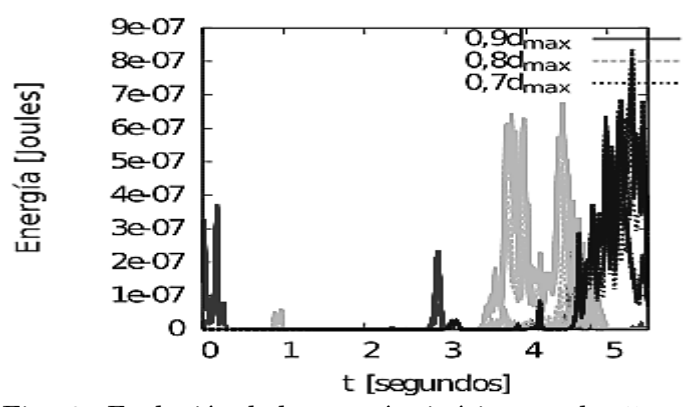

Fig. 9. Evolución de la energía cinética con $d_{50}=5 \mathrm{~mm}$. Fuente: Autores.

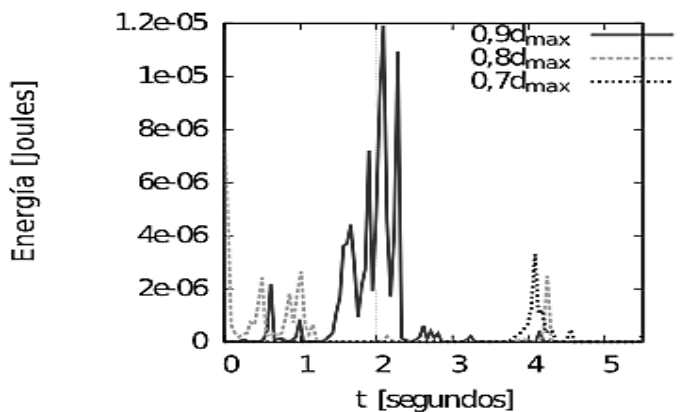

Fig. 10. Evolución de la energía cinética con $\mathrm{d}_{50}=10 \mathrm{~mm}$. Fuente: Autores.

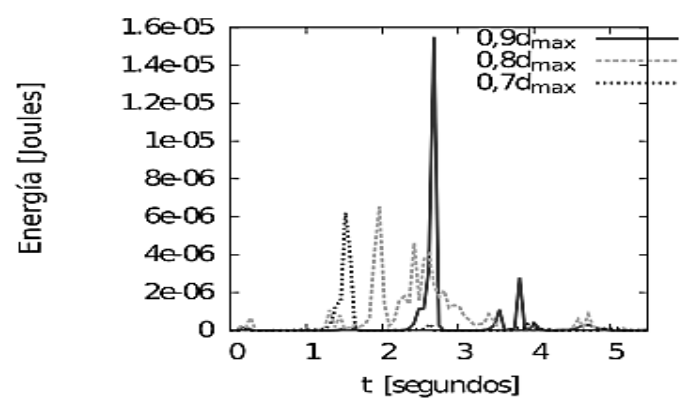

Fig. 11. Evolución de la energía cinética con $\mathrm{d}_{50}=15 \mathrm{~mm}$. Fuente: Autores.

\section{2 Ángulo máximo}

El ángulo máximo o ángulo de fricción externa es el ángulo en el cual las partículas del material desagregado comienzan a deslizarse. El valor de este ángulo es bastante cercano al ángulo de reposo [30]. La importancia de esta variable es la capacidad de capturar información desde la geomorfología, para establecer zonas con mayor potencialidad de generación de flujo de detritos. Wu y Sun [30] concluyen que, con el incremento del tamaño de la partícula, el ángulo de reposo disminuye y decrece rápidamente para tamaños pequeños. El mismo comportamiento se puede ver en las Fig. 12, Fig. 13 y Fig. 14; no obstante, se observa que para el valor de $\mathrm{d} 50=10 \mathrm{~mm}$ el ángulo se ve afectado ligeramente. En el caso contrario, los valores extremos de d50 son los más afectados por el $\mathrm{Cu}$ en una diferencia no mayor a $5^{\circ}$. Se observa que la disminución del dmax parece tener pocos efectos sobre el ángulo máximo. 


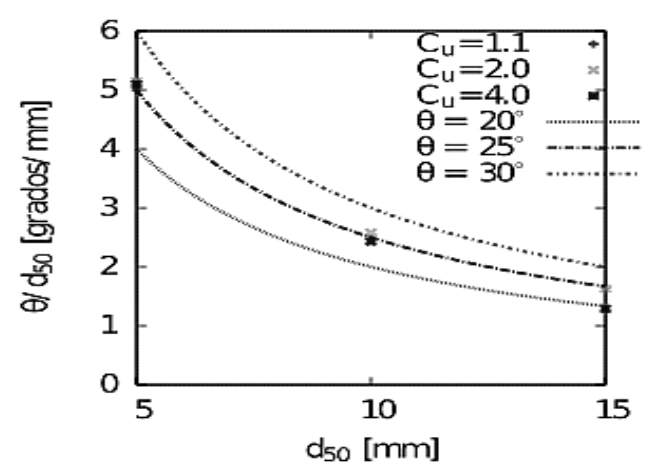

Fig. 12. Ángulo máximo alcanzado con $0.9 \mathrm{~d}_{\max }$. Fuente: Autores.

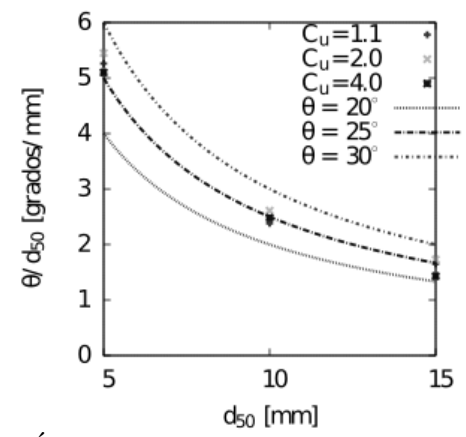

Fig. 13. Ángulo máximo alcanzado con 0.8 dmax. Fuente: Autores.

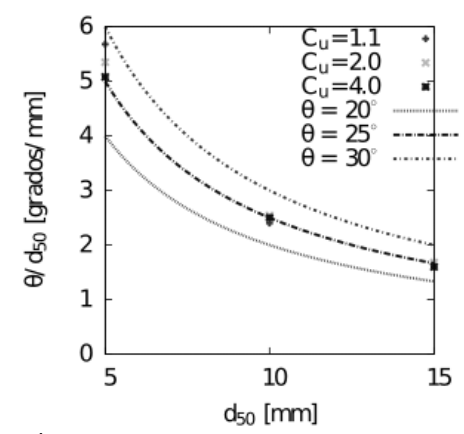

Fig. 14. Ángulo máximo alcanzado con 0.7 dmax. Fuente: Autores.

\subsection{Energía cinética}

La capacidad de daño de un flujo de detritos está asociada a procesos altamente energéticos [2], energía que está relacionada directamente con la masa movilizada.

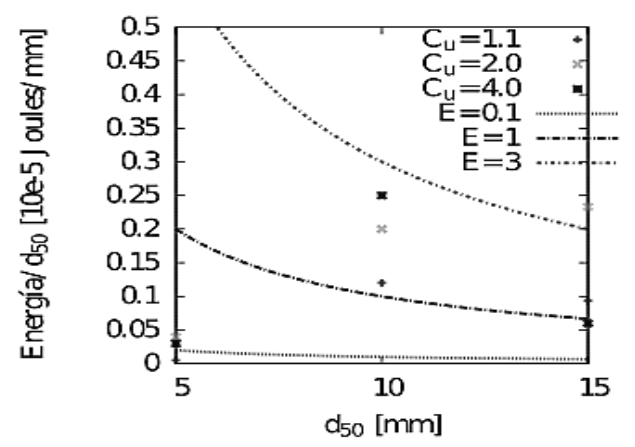

Fig. 15. Energía cinética máxima en los procesos generados con $0.9 d_{\max }$. Fuente: Autores.

De acuerdo con los resultados (ver Fig. , Fig. 16 y Fig. 17), se observa que con el incremento de $\mathrm{d}_{50}$, la energía aumenta por efecto de escala que se traduce en mayor masa. Por otra parte, las muestras polidispersas tienden a reportar valores mayores de energía. Como se sabe, una muestra polidispersa puede contener una relación de vacíos menor y por lo tanto una mayor densidad.

Valores pequeños de $d_{\max }$ concentran valores de energía en menores umbrales, debido a que las partículas de mayor tamaño son reemplazadas por varias de un tamaño menor, generándose más vacíos y por lo tanto menor masa movilizada.

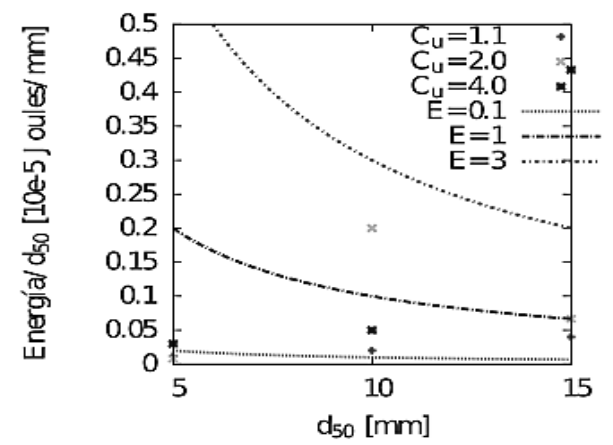

Fig. 16. Energía cinética máxima en los procesos generados con $0.8 d_{\max }$. Fuente: Autores. 


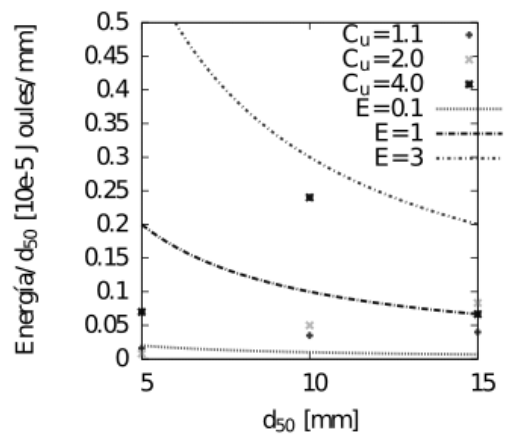

Fig. 17. Energía cinética máxima en los procesos generados con $0.7 d_{\max }$. Fuente: Autores.

\subsection{Profundidad de flujo}

La importancia de esta variable radica en que está relacionada con la energía cinética, pues una profundidad de flujo $\left(\mathrm{F}_{\mathrm{d}}\right)$ mayor representa una masa movilizada mayor. De las modelaciones realizadas se obtuvo que las muestras con un coeficiente de uniformidad menor presentan planos de debilidad con mayor frecuencia. En consecuencia, su profundidad de falla se puede localizar superficialmente, caso contrario a lo que ocurre en una muestra polidispersa. Es de esperar que la profundidad de flujo sea menor para valores menores de $\mathrm{d}_{50}$. Para ilustrar, en el caso donde el proceso se genere en la condición más superficial en una muestra monodispersa, la profundidad de flujo sería igual al $\mathrm{d}_{50}$, es decir que la $\mathrm{F}_{\mathrm{d}}$ crecerá en igual cantidad que el valor medio de la partícula. Sin embargo, esta tendencia es difícil mantener en suelos donde existen diversos tamaños de partículas.

Finalmente, se muestra que un $d_{\max }$ menor tiende a disminuir y concentrar la profundidad de flujo por cada $\mathrm{d}_{50}$ evaluado (ver Fig. 18, Fig. 19 y Fig. 20). Las partículas de mayor tamaño controlan la profundidad de flujo ampliamente, pues la superficie de falla no puede pasar a través de la partícula y por lo tanto pasa por debajo o por encima de ella.

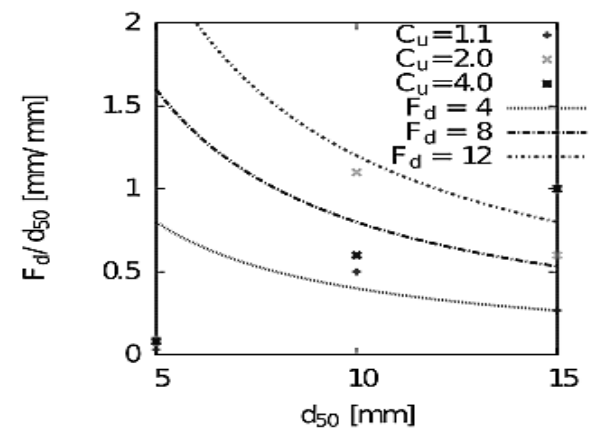

Fig. 18. Profundidad de flujo promedio para un $0.9 d_{\max }$. Fuente: Autores.

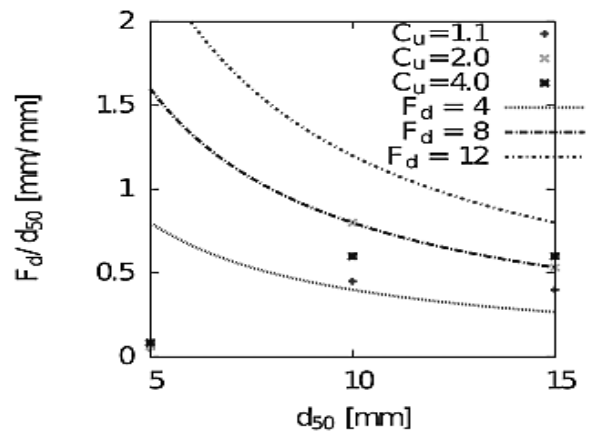

Fig. 139. Profundidad de flujo promedio para un $0.8 d_{\max }$. Fuente: Autores.

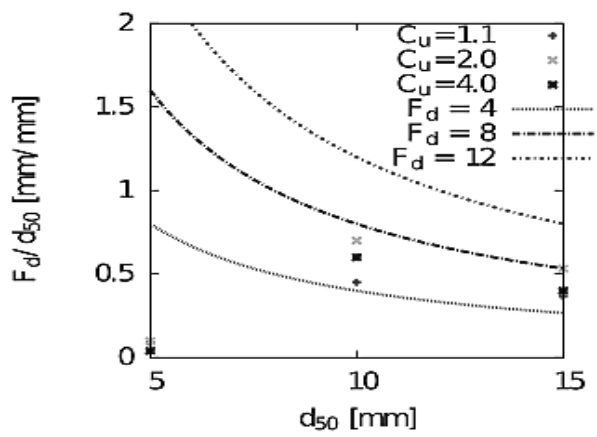

Fig.20. Profundidad de flujo promedio para un $0.7 \mathrm{dmax}$. Fuente: Autores.

\section{CONCLUSIONES}

Los resultados muestran una variación no mayor a $5^{\circ}$ del ángulo máximo alcanzado bajo el análisis paramétrico, siendo menor la dispersión en el valor intermedio del $d_{50}(10 \mathrm{~mm})$. Sin embargo, el tamaño medio de la partícula $\left(\mathrm{d}_{50}\right)$ tiende a controlar la duración del proceso de remoción en masa evaluado. Se involucra un menor tiempo en el proceso a medida que el ta- 
maño medio de la partícula aumenta. Aunque el tiempo del proceso es menor, la energía máxima desarrollada es mayor pues los valores de $\mathrm{d}_{50}$ más altos reportan crecimiento en las dos variables que se encuentran estrictamente relacionadas: la energía cinética y profundidad de flujo.

Aunque el valor de diámetro medio de la partícula es el parámetro que más influencia tiene sobre en el comportamiento

\section{REFERENCIAS}

[1] P. A. Cundall and O. D. L. Strack, "A discrete numerical model for granular assemblies," Géotechnique, vol. 29, no. 1, pp. 47-65, Mar. 1979.

[2] M. Jakob, O. Hungr, and D. M. Jakob, Debris-flow hazards and related phenomena, vol. 739. Springer, 2005.

[3] L. S. Blake and E. H. Probst, Civil engineer's reference book. Newnes-Butterworths., 1975.

[4] J. Suarez Díaz, Deslizamientos y estabilidad de taludes en zonas tropicales. Edición UIS, 1998.

[5] D. Cornforth, Landslides in practice: investigation, analysis, and remedial/preventative options in soils. Wiley, 2005.

[6] M. A. Maidana, M. E. Infantes, J. B. Lorente, M. y A. Departamento de Inginiería Hidráulica, and U. P. de C. Laboratorio de Inginiería Marítima, "Desarrollo de un modelo numérico 3D en elementos finitos para las ecuaciones de Navier-Stokes: aplicaciones oceanográficas," Universidad Politécnica de Cataluña, 2007.

[7] C. O'Sullivan, Particulate discrete element modelling. Taylor \& Francis, 2011.

[8] A. Phillip Grima and P. Wilhelm Wypych, "Discrete element simulations of granular pile formation," Eng. Comput., vol. 28, no. 3, pp. 314-339, Apr. 2011.

[9] P. Gajjar, K. van der Vaart, A. R. Thornton, C. G. Johnson, C. Ancey, and J. M. N. T. Gray, "Asymmetric breaking size-segregation waves in dense granular free-surface flows," J. Fluid Mech., vol. 794, pp. 460-505, May 2016.

[10] A. Leonardi, F. K. Wittel, M. Mendoza, R. Vetter, and H. J. Herrmann, "Particle-FluidStructure Interaction for Debris Flow Impact on Flexible Barriers," Comput. Civ. Infrastruct. Eng., vol. 31, no. 5, pp. 323-333, de la muestra, se evidencia que efectos sobre el flujo de detritos ocasionados por coeficiente de uniformidad y tamaño máximo del material son igualmente relevantes. El primero reporta un aumento en la energía cinética y profundidad de flujo cuando la muestra es bien gradada. El último, a medida que disminuye, reduce tanto la energía cinética producida como la profundidad de flujo, confinándolos en umbrales bajos.

[11] C. Y. Lu, C. L. Tang, Y. C. Chan, J. C. Hu, and C. C. Chi, "Forecasting landslide hazard by the 3D discrete element method: A case study of the unstable slope in the Lushan hot spring district, central Taiwan," Eng. Geol., vol. 183, pp. 14-30, Dec. 2014.

[12] Q. Deng, L. Gong, L. Zhang, R. Yuan, Y. Xue, X. Geng, and S. Hu, "Simulating dynamic processes and hypermobility mechanisms of the Wenjiagou rock avalanche triggered by the 2008 Wenchuan earthquake using discrete element modelling," Bull. Eng. Geol. Environ., pp. 1-14, Jul. 2016.

[13] B. Chareyre, D. Marzougui, and J. Chauchat, "Can we reduce debris flow to an equivalent one-phase flow?," IOP Conf. Ser. Earth Environ. Sci., vol. 26, no. 1, p. 12009, Sep. 2015.

[14] S. Longo and A. Lamberti, "Grain shear flow in a rotating drum," Exp. Fluids, vol. 32, no. 3, pp. 313-325, Mar. 2002.

[15] R. Y. Yang, A. B. Yu, L. McElroy, and J. Bao, "Numerical simulation of particle dynamics in different flow regimes in a rotating drum," Powder Technol., vol. 188, no. 2, pp. 170-177, Dec. 2008.

[16] G. G. D. Zhou and C. W. W. Ng, "Numerical investigation of reverse segregation in debris flows by DEM," Granul. Matter, vol. 12, no. 5, pp. 507-516, Oct. 2010.

[17] J. R. Third, D. M. Scott, S. A. Scott, and C. R. Müller, "Tangential velocity profiles of granular material within horizontal rotating cylinders modelled using the DEM," Granul. Matter, vol. 12, no. 6, pp. 587-595, Dec. 2010.

[18] S.-C. Hsu, C.-H. Jaing, and N.-C. Chen, "Modeling of Debris Flow Using Distinct Element Method," in IACGE 2013, 2013, pp. 713-720.

[19] R. Chand, M. A. Khaskheli, A. Qadir, B. Ge, and $\mathrm{Q}$. Shi, "Discrete particle simulation of radial segregation in horizontally rotating drum: Effects of drum-length and non- 
rotating end-plates," Phys. A Stat. Mech. its Appl., vol. 391, no. 20, pp. 4590-4596, Oct. 2012.

[20] J. Xu, H. Qi, X. Fang, L. Lu, W. Ge, X. Wang, M. Xu, F. Chen, X. He, and J. Li, "Quasireal-time simulation of rotating drum using discrete element method with parallel GPU computing," Particuology, vol. 9, no. 4, pp. 446-450, Aug. 2011.

[21] X. Liu, W. Ge, Y. Xiao, and J. Li, "Granular flow in a rotating drum with gaps in the side wall," Powder Technol., vol. 182, no. 2, pp. 241-249, Feb. 2008.

[22] H. T. Chou, C. F. Lee, Y. C. Chung, and S. S. Hsiau, "Discrete element modelling and experimental validation for the falling process of dry granular steps," Powder Technol., vol. 231, no. 0, pp. 122-134, Aug. 2012.

[23] P. Y. Liu, R. Y. Yang, and A. B. Yu, "DEM study of the transverse mixing of wet particles in rotating drums," Chem. Eng. Sci., vol. 86, no. 0, pp. 99-107, Feb. 2013.

[24] K. L. Johnson, Contact mechanics, 1st ed. Cambridge university press, 1987.

[25] H. D. R. D. Mindlin, "Elastic spheres in contact under varying oblique forces," $J$.
Appl. Mech., vol. 20, pp. 327-344, 1953.

[26] A. A. Serrano and J. M. Rodríguez-Ortíz, "A contribution to the mechanics of heterogeneous granular media.," 2013.

[27] J. M. Rodríguez-Ortíz, "Estudio del comportamiento de medios granulares heterogéneos mediante modelos discontinuos analógicos y matemáticos," Universidad Politécnica de Madrid, 1974.

[28] C. O'Sullivan and J. D. Bray, "Selecting a suitable time step for discrete element simulations that use the central difference time integration scheme," Eng. Comput., vol. 21, no. 2/3/4, pp. 278-303, Mar. 2004.

[29] T. Belytschko, B. Moran, and W. K. Liu, Nonlinear finite element analysis for continua and structures, vol. 1. Wiley, 1999.

[30] A. Wu, Y. Sun, and X. Liu, Granular dynamic theory and its applications. Springer, 2008.

[31] R. B. Canelas, A. J. C. Crespo, J. M. Domínguez, R. M. L. Ferreira, and M. Gómez-Gesteira, "SPH-DCDEM model for arbitrary geometries in free surface solidfluid flows," Comput. Phys. Commun., vol. 202, pp. 131-140, May 2016. 